\title{
Effectiveness of prenatal screening for Down syndrome on the basis of maternal age in Cape Town
}

\author{
M F Urban, C Stewart, T Ruppelt, L Geerts
}

Objective. The prenatal screening programme for Down syndrome (DS) in the South African public health sector remains primarily based on advanced maternal age (AMA). We assessed the changes over time and effectiveness of this screening programme within a Cape Town health district.

Methods. Retrospective analysis of the Groote Schuur Hospital Cytogenetic Laboratory and Pregnancy Counselling Clinic databases and audit of maternal delivery records at a primary health care facility.

Results. The number of amniocenteses performed for AMA in consecutive 5-year periods reduced progressively from 786 in 1981 - 1985 to 360 in 2001 - 2005. Comparing prenatal with neonatal diagnoses of DS, the absolute number and the proportion diagnosed prenatally have remained relatively constant over time.
The Pregnancy Counselling Database showed that, of 507 women receiving genetic counselling for AMA in 2008 - 2009, 158 (31.1\%) accepted amniocentesis - uptake has reduced considerably since the early 1990s. The audit of women delivering at a primary care facility found that only $10(16.4 \%)$ of 61 AMA women reached genetic counselling in tertiary care: reasons included late initiation of antenatal care and low referral rates from primary care.

Conclusion. Prenatal screening and diagnosis for DS based on AMA is working ineffectively in the Cape Town health district assessed, and this appears to be representative of a broader trend in South Africa. Inclusion of fetal ultrasound in the process of prenatal screening for DS should be explored as a way forward.

S Afr Med J 2011;101: 45-48.
Down syndrome (DS) is the most common genetic cause of intellectual disability. Evidence suggests that live-birth prevalence of DS is high in South Africa: 1.33 - 1.8 per 1000 live births in two urban areas, ${ }^{1,2}$ and 2.1 per 1000 in a rural area. ${ }^{3}$

Prenatal screening for DS was established in the 1970s using advanced maternal age (AMA) as a risk factor, and this remains the standard of care in the public health sector of South Africa. National policy provides for free amniocentesis to be offered to AMA women, whereas biochemical screening is not offered and access to fetal ultrasound is limited.

Cape Town's public health services are among the best developed in South Africa. Although access to fetal ultrasound has increased in recent years, AMA remains a frequent indication for prenatal genetic counselling. The study focused on the Cape Town Metropole West health district (CT West) to obtain evidence regarding the effectiveness of age-based screening for the prevention of DS, and preliminary evidence regarding factors affecting utilisation of prenatal diagnosis. We assessed (i) laboratory trends in prenatal diagnostic testing over

Division of Human Genetics, University of Cape Town

M F Urban, MB BCh, BSc (Med), FCPaed (SA), MMed (Paed), Cert (Med Genet)

Fetal Medicine Unit, Department of Obstetrics and Gynaecology, Groote Schuur Hospital and University of Cape Town C Stewart, MB ChB, FCOG, MMed (O\&G)

Cytogenetic Laboratory, National Health Laboratory Service, Groote Schuur Hospital T Ruppelt, NDip, BTech

Fetal Medicine Clinic, Department of Obstetrics and Gynaecology, Tygerberg Hospital and Stellenbosch University

L Geerts, MB ChB, MRCOG, Dip (Fetal Med), HonsBSc (Hum Genet) a 30-year period, (ii) trends in uptake of prenatal diagnostic testing among women receiving genetic counselling and (iii) the referral pathway from primary care to tertiary care.

\section{Methods}

Study population. Women attending state antenatal health care services in CT West, which comprises a large and diverse urban population, with a majority coloured population, and minorities of whites and blacks. The district has experienced rapid urbanisation and considerable demographic changes. In 2005, there were approximately 34000 deliveries in the district (Patel, unpublished data).

Study sites and services. The antenatal health service and cytogenetic laboratory service in CT West have been stable since the 1970s. The health service includes several primary and secondary level facilities, and tertiary level fetal medicine and pregnancy counselling services at Groote Schuur Hospital (GSH). The University of Cape Town (UCT) Cytogenetic Laboratory (CL) has provided prenatal and postnatal diagnostic laboratory services to CT West throughout the period. Prenatal diagnostic testing is almost exclusively by amniocentesis, and indications have included increased risk of DS (most commonly AMA, but more recently also based on ultrasound markers (first trimester nuchal translucency thickness and second trimester soft markers) and fetal anomalies detected on ultrasound. The age cut-off for AMA counselling has varied over the years.

UCT CL database. The laboratory has maintained a database of samples received since the early 1970s. Initially this was a written record but all records were transferred to Microsoft Access in the mid-1990s, and a parallel written and computerised database was maintained. To improve data quality, the database was cleaned by cross-referencing blank or unclear computer data fields with the written records - the remaining missing data represented less than $5 \%$ of data points per category. Categories analysed were: type of sample received, maternal age, indication for testing, referring institution (as a proxy for the patient's residential address) and the karyotype result obtained. Data from January 1976 to December 2005 were retrospectively analysed in 5-year periods to assess trends in prenatal and postnatal diagnosis of autosomal trisomies. The information did not permit us to link prenatal and postnatal diagnoses. Since the 
laboratory handles all prenatal and postnatal tests for the CT West district, we restricted the analysis to samples emanating from this area to maximise comparability of prenatal and postnatal samples. Neonatal samples (from the first 28 days of postnatal life) were analysed separately from other postnatal samples, because prenatal and neonatal diagnoses are most likely to relate to the same cohort of pregnancies. We accepted that under-ascertainment in the neonatal period would probably lead to some overestimation of the proportion of DS cases diagnosed prenatally.

GSH Prenatal Counselling Clinic database. Since January 2008, a Pregnancy Counselling database (Microsoft Access) has been maintained for clinical audit of genetic counselling sessions including: information on maternal age, gestational age, indication for counselling, ultrasound results, uptake and outcome of prenatal diagnostic testing. This was retrospectively analysed from January 2008 to December 2009, to assess the uptake of prenatal diagnostic testing for different indications.

Primary care facility. To explore the referral process from primary care to the prenatal genetic counselling service, a single, well-functioning primary care facility in the CT Metro West district - the Hanover Park Maternity Obstetric Unit - was audited. This comprised a retrospective record review of consecutive antenatal records of AMA women who delivered at the facility between 1 January 2009 and 30 June 2009.

Statistical analysis. Where relevant, univariate analysis was performed using the chi-square test.

Ethical considerations. The cytogenetic and pregnancy counselling databases are maintained for record-keeping and clinical audit. Their design and maintenance ensure patient confidentiality in accordance with UCT Research Ethics Committee recommendations. The audit of the primary care facility was approved by the UCT Research Ethics Committee and the relevant sub-district of the provincial Department of Health.

\section{Results}

In the study period, 5943 amniotic fluid samples were tested in the UCT CL, of which 5319 were from women referred from health care facilities in the now designated CT West. Fig. 1 shows the trend in the number of amniocentesis samples processed, together with their indication. The total number peaked in the early 1980s and then decreased, with a plateau beyond 1996. There has been a continuing reduction in amniocenteses for AMA despite a concurrent reduction in the cut-off age for offering AMA-based testing. The number of amniocenteses performed for AMA more than halved from a high of 786 in 1981 - 1985, to a low of 360 in $2001-2005$.

Fig. 2 shows the number of DS diagnoses made in the prenatal period, neonatal period, and all postnatal diagnoses. The total

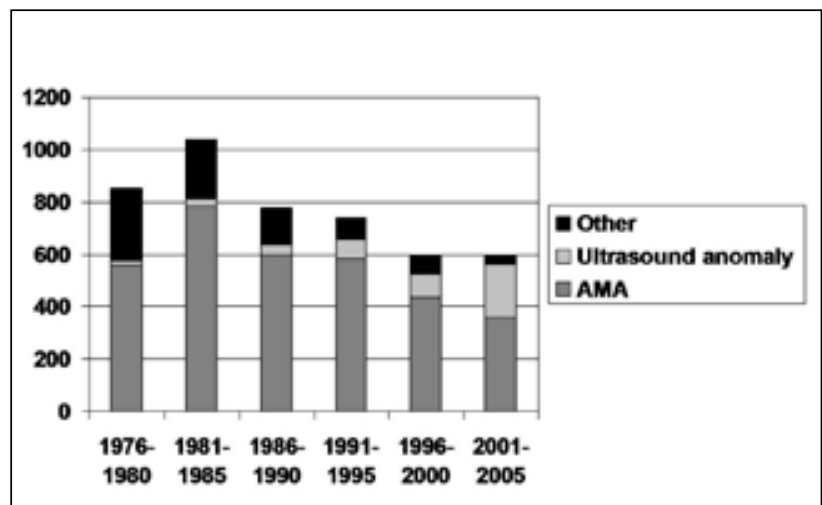

Fig. 1. Uptake of amniocentesis by indication, and per 5-year period.

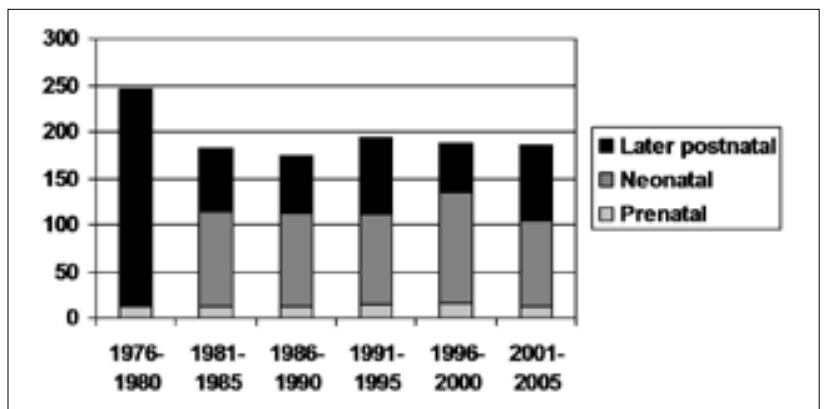

Fig. 2. Frequency of prenatal and postnatal diagnosis of DS per 5-year period.

number of DS cases detected in the neonatal period, or at any age postnatally, has remained relatively constant except for the first 5-year period (probably representing a catch-up in karyotype testing in this period). Prenatal diagnoses have also remained relatively constant, but only a small proportion of DS cases were diagnosed prenatally in any time period. Using as a denominator the DS cases diagnosed by the end of the neonatal period (per 5-year interval), the proportion of the cases diagnosed prenatally varied from a minimum of $10.4 \%$ ( 12 of 115 cases) to a maximum of $13.5 \%$ (15 of 111 cases). In the most recent 5-year period, 13 (12.5\%) of 104 cases were diagnosed prenatally - the indication for testing was AMA in 7 of these cases, and related to ultrasound findings in 6. Alternatively, using total DS diagnoses at any age as a denominator, the proportion of prenatal diagnoses varied from $6.6 \%$ (12 of 183) to $8.6 \%$ (16 of 187).

Uptake of amniocentesis by women receiving genetic counselling for AMA or for fetal anomalies is shown in Table I, and is presented alongside data from the same service for a 2-year period in 1992 1994. During the intervening years, the cut-off age for referral to genetic counselling was reduced from 39 to 37 years. Comparing the two time periods, the uptake of amniocentesis for AMA more than halved, even if the analysis was restricted to women $>39$ years. In 2008 - 2009, the uptake for the indication of AMA was significantly lower than that for fetal anomalies $(p<0.0001$, odds ratio (OR) 0.21 $(0.14-0.30))$.

The primary care facility audit (Fig. 3) shows dropout at several steps on the referral pathway of women from primary care to prenatal diagnosis in tertiary care. Less than half of women presenting before 20 weeks' gestation received genetic counselling. The reasons for this are uncertain, but clinic midwives indicated that there was uncertainty about the age cut-off for referral. Although provincial referral guidelines recommend referral for amniocentesis at $16-20$ weeks for women $>37$ years old, some nurses still used 38 years as a cut-off (a previous guideline recommendation). The audit numbers

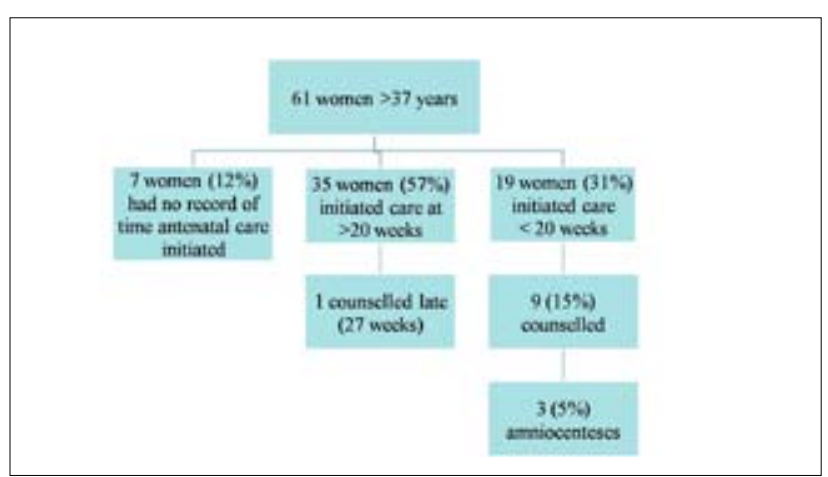

Fig. 3. Flow diagram of AMA patients from primary health care through the referral process for genetic counselling. 
Table I. Uptake of amniocentesis per indication, in Cape Town Metropole West - comparison of two time periods

\begin{tabular}{|c|c|c|c|}
\hline & 1992-1994 (2-year period $)^{*}$ & 2008-2009 (2-year period) & $p$-value (Yates chi square) \\
\hline Total & 466 & 1185 & \\
\hline Amniocentesis uptake in all AMA women & $191 / 258(74.0 \%)^{\dagger}$ & $158 / 507(31.1 \%)^{\ddagger}$ & $\begin{array}{l}p<0.0001 \\
\text { OR } 0.16(\text { CI } 0.11-0.22)\end{array}$ \\
\hline Amniocentesis uptake in women $>39$ years & $191 / 258(74.0 \%)$ & $123 / 360(34.1 \%)$ & $\begin{array}{l}p<0.0001 \\
\text { OR } 0.18(\text { CI } 0.13-0.26)\end{array}$ \\
\hline Fetal anomaly on ultrasound & $N=66$ & $N=399$ & \\
\hline No. offered amniocentesis & Not documented & 172 & two \\
\hline Uptake of amniocentesis & 17 & $\begin{array}{l}118 / 172(68.6 \% \text { of those offered } \\
\text { amnio) }\end{array}$ & \\
\hline Other & 142 & 279 & \\
\hline $\begin{array}{l}{ }^{*} \text { Data extracted from Viljoen et al. } \\
{ }^{4} \text { In this time period, the age cut-off for AMA was } 39 \text { years. } \\
{ }^{*} \text { In this time period, the age cut-off for AMA was } 37 \text { years. }\end{array}$ & & & \\
\hline
\end{tabular}

were insufficient to assess whether this significantly reduced genetic counselling in 37-year-olds. The midwives also thought that women preferred referral for ultrasound rather than for age-related reasons.

\section{Discussion}

The main findings of the study are that, in CT West, $(i)$ only a small fraction of proven DS cases are diagnosed prenatally, (ii) utilisation of AMA counselling is low, and (iii) uptake of prenatal testing for AMA has reduced considerably over time.

The proportion of Down syndrome cases diagnosed prenatally has remained low and relatively constant over time, with an increasing proportion of these diagnoses based on ultrasound-detected fetal anomalies rather than AMA screening. The total number of postnatal DS diagnoses in CT West has also remained relatively constant despite rapid urbanisation and increasing numbers of births in the district. We speculate that this may be related to a reduction in the average age at which women have children.

Audit data obtained from laboratories providing cytogenetic services to the government sector indicates a nationwide low utilisation of prenatal diagnostic testing for AMA. In 2008, these laboratories received 529 amniocenteses for AMA (unpublished data for 6 of 7 national laboratories provided by T Lane, G de Jong, S Kavonic, W Pfaffenzeller, T Ruppelt). Since there were approximately 830000 deliveries in the government health sector of South Africa in $2007,{ }^{5}$ and an estimated $17.2 \%$ of births are to women aged $>35$ years, ${ }^{6}$ it is clear that only a tiny minority of AMA women receive prenatal diagnostic testing. Four of the 9 provinces of South Africa have no tertiary fetal medicine and medical genetic services.

The primary care facility audit gives possible reasons for low rates of genetic counselling for AMA in our service: late initiation of antenatal care and missed opportunities for prenatal counselling in AMA women attending early, both owing to patient- and staffrelated factors. The uncertainty among primary care staff regarding current guidelines for referral to genetic counselling suggests a need for clear communication of provincial policy documents to primary care level. Late initiation of antenatal care is common in South Africa; it is estimated ${ }^{7}$ that only $27 \%$ of South African women initiate antenatal care before 20 weeks' gestation. South African studies have found several reasons for late initiation of antenatal care, including: (i) socio-economic factors e.g. related to transport costs, (ii) lack of knowledge of the importance of antenatal care, (iii) unwelcoming health services, ${ }^{8}$ (iv) lack of knowledge of the potential health threats in pregnancy, ${ }^{9}$ and $(v)$ frequent missed opportunities for referral to genetic counselling from other primary care services - either clinics or private sector general practitioners. ${ }^{10}$ One or more of these factors are probably at work in our setting, and further study is warranted.

A dramatic reduction in the uptake of amniocentesis for AMA in CT West in the last 15 years is demonstrated. The reasons are uncertain, but several possibilities are outlined. Significant shifts have occurred in the demographic characteristics of women using public sector antenatal services: higher-income women now attend private sector antenatal care, which may be important since women of higher socio-economic status are more likely to accept amniocentesis. ${ }^{11}$ The democratic culture since 1994 has significantly increased public awareness of 'patient rights', which might have influenced reproductive choices. Public perceptions of disability might have changed for several reasons, including the introduction of a grant for the care of disabled children. There has been a dramatic increase in HIV among South African women, and untreated HIVinfected South African women are less likely to want amniocentesis. ${ }^{12}$ However, HIV is at most a partial explanation: the availability of antiretroviral prophylaxis has improved considerably in recent years; and in addition, the estimated $15.3 \%$ prevalence of HIV among pregnant women in the Western Cape $^{13}$ appears insufficient to have had a major impact on overall amniocentesis rates.

Amniocentesis uptake for AMA is variable in other parts of South Africa: $29.9 \%$ in Johannesburg ( $\mathrm{T}$ Wessels, unpublished data), and 52.2\% in Cape Town Metropole East district ( $\mathrm{G}$ de Jong, unpublished data). Reasons for these differences have not been explored but may include differences in culture, access, counselling and referral patterns.

The reduction in amniocentesis for AMA has been offset by an increasing number of amniocenteses for fetal anomalies, resulting in a levelling off in overall numbers of amniocenteses performed. In part, these changes reflect an improvement in the availability and quality of prenatal ultrasound, with an emphasis mainly on the 20 22 -week fetal anomaly scan. It is uncertain whether this has affected referral patterns for AMA.

It is clear that many AMA women do not receive genetic counselling and many others decline prenatal testing, and that the strategy of AMA screening is inefficient compared with other prenatal screening methods. A local study found that an age cut-off of 37 years had 
a maximal detection rate of $43.3 \%$ and that lowering the cut-off to 35 years would only improve the detection rate to $52 \%$, while significantly increasing the number of procedures performed. ${ }^{14}$

The way forward, we believe, is that prenatal screening and diagnosis should continue to be offered, but that alternatives to agebased screening must be sought. A promising option would be to improve integration of DS screening with fetal ultrasound services. This would permit screening for DS in younger women as well, and could be integrated with screening for other fetal anomalies. An ultrasound-based approach achieved a prenatal detection rate for DS of $25.3 \%{ }^{14}$ - approximately double our maximum estimated detection rate. While the utility of ultrasound for prenatal screening and diagnosis has been debated, ${ }^{15}$ ultrasound has additional benefits, including accurate pregnancy dating and early detection of unexpected problems. ${ }^{16,17}$ Fetal ultrasound also appears to be popular with parents and primary health care providers, which will probably improve the link between primary and tertiary care. Fetal ultrasound services are therefore increasingly used and, in metropolitan areas, fetal medicine is increasingly important. The service should be interdisciplinary, including fetal medicine specialists, ultrasonographers, medical geneticists and genetic counsellors, and should aim to provide an equitable service at all levels of care, and to women outside metropolitan areas.

Limitations. The study was limited by the retrospective nature of the data, although this factor applies particularly to the analysis of the cytogenetic laboratory database since it represents 30 years of historical data. The available data did not permit us to link prenatal and postnatal diagnoses of DS, and our approaches to improving the comparability of prenatal and postnatal diagnoses are discussed in the methods section. Unfortunately, no detailed annual provincial or national data exist regarding maternal age, which could be used to estimate the expected birth prevalence of DS, and would provide useful comparative information. For convenience, the audit of the primary health care facility focused on women delivering at the facility, and excluded women at high obstetric risk who would be transferred to other levels of care. Therefore the sample represents only AMA women of lower obstetric risk. These weaknesses are mitigated by the multiple data sources used and similar trends from various sources.

We thank Ms B Mentoor for collecting data at the primary health centre, and the staff of the primary health centre for their participation.

\section{References}

1. Delport SD, Christianson AL, van den Berg HJS, Wolmarans L, Gericke GS. Congenital anomalies in black South African liveborn neonates at an urban academic hospital. S Afr Med J 1995;85:11-15.

2. Christianson AL, Kromberg JGR. Maternal non-recognition of Down syndrome in black South African infants. Clin Genet 1996;49:141-144.

3. Venter PA, Christianson AL, Hutamo CM, Mahura MP, Gericke GS. Congenital anomalies in rural black South African neonates - a silent epidemic? S Afr Med J 1995;85:15-20.

4. Viljoen D, Oosthuizen C, van der Westhuizen S. Patient attitudes to prenatal screening and termination of pregnancy at Groote Schuur Hospital: a two year prospective study. East Afr Med J 1996;73:327329.

5. Pattinson RC, ed. Saving Babies 2006-2007: Sixth Perinatal Care Survey of South Africa. Pretoria: Tshepesa Press, 2009.

6. United Nations. Demographic Yearbook Series. New York: United Nations, 2003.

7. Pattinson RC, Etsane E, Snyman JS, et al. Report to UNICEF on the scaling-up of the Basic Antenatal Care quality improvement programme in two sub-districts per province in South Africa (Draft). Pretoria: MRC Maternal and Infant Health Care Strategies Research Unit and the University of Pretoria, Preto

8. Abrahams $\mathrm{N}$, Jewkes R, Mvo Z. Health care-seeking practices of pregnant women and the role of the midwife in Cape Town, South Africa. J Midwifery Womens Health 2001;46:240-247.

9. Myer L, Harrison A. Why do women seek antenatal care late? Perspectives from rural South Africa. J Myer L, Harrison A. Why do women seek ant

Midwifery Womens Health 2003;48:268-272.
. Watcham SJ, Schön S, Christianson AL. Neglect in the care of pregnant South African women of advanced maternal age. S Afr Med J 2007;97:1064-1069.

11. Julian-Reynier C, Macquart-Moulin G, Moatti JP, Aurran Y, Chabal F, Aymé S. Reasons for women's non-uptake of amniocentesis. Prenat Diagn 1994;14(9):859-864.

12. Bee J. The influence of HIV status on women of advanced maternal age presenting for genetic counseling [Research report]. Johannesburg: Division of Human Genetics, University of the Witwatersrand, 2005.

13. Dorrington R, Bourne D. Re-estimated provincial HIV antenatal survey prevalence for 2007 and a reinterpretation of the national trend. S Afr Med J 2008;98(12):940-941.

14. Geerts L. Prenatal diagnosis of chromosomal abnormalities in a resource-poor setting. Int J Gynecol Obstet 2008;103:16-21.

15. Neilson JP. Ultrasound for fetal assessment in early pregnancy. Cochrane Database Syst Rev (2):CD000182, 2000.

16. Geerts L, Theron AM, Grove D, Theron GB, Odendaal HJ. A community-based obstetric ultrasound service. Int J Gynecol Obstet 2004;84:23-31.

17. van Dyk B, Motto JA, Buchmann EJ. Routine second-trimester ultrasound for low risk pregnancies in a South African community. Int J Gynecol Obstet 2007;98:257-258.

Accepted 17 August 2010. 